J. of Electromagn. Waves and Appl., Vol. 16, No. 1, 129-143, 2002

\title{
RESONANT REFLECTION FROM DIPOLE ARRAYS LOCATED VERY NEAR TO CONDUCTING PLANES
}

\author{
P. A. Belov and S. A. Tretyakov \\ Radio Laboratory \\ Helsinki University of Technology \\ P.O. Box 3000, FIN-02015 HUT, Finland
}

\begin{abstract}
Plane-wave reflection from regular arrays of small particles positioned very near to an ideally conducting plane is theoretically studied. It is found that extremely thin (in wavelength) coverings with resonant properties can be realized if the array particles are small dipole antennas loaded by bulk inductances. This offers a possibility to realize an artificial magnetic conductor as a thin covering over conducting bodies. Dependencies of the resonance frequency and the resonant curve width on geometrical parameters are given. The theory is supported by numerical examples.
\end{abstract}

\section{Introduction}

2 Reflection from a Dipole Array Near a Metal Plane

2.1 Reflection Theory

2.2 Interaction Constant

\section{Resonance Condition}

3.1 Point Dipoles

3.2 Spherical Particles

3.3 Arrays of Short Loaded Wire Antennas

4 Numerical Example

5 Conclusion

\section{References}




\section{INTRODUCTION}

Recently, much attention was paid to novel thin artificial impedance surfaces for applications in antennas and microwave devices [1]. For applications it is important that the thickness of the layer be as small as possible. One possible realization [1] is based on the use of quasibulk cells, so that the resonant frequency is lowered in thin layers due to concentration of electric fields in equivalent capacitances. However, the geometry of these layers is quite complicated (3D cells), and it is not possible to increase the surface inductance keeping the thickness small. Another possible cause for a resonance response can be local reactive fields generated at inhomogeneities [2] or cracks [3].

In this paper we propose a new idea to create thin resonant coverings for conducting surfaces making use of doubly periodic array of small resonant scatterers positioned close to an ideally conducting plane. It is shown that such a structure at frequencies near the resonance is described by a very high reactive surface impedance and behaves as a magnetic screen.

Let us consider an ideally conducting plane illuminated by a normally incident plane wave. Suppose we want to considerably modify the electromagnetic response of this plane covering the surface by a very thin (compared to the wavelength) non-magnetic layer. In particular, we are interested in creating artificial magnetic surfaces in such a way. At first glance, this seems to be impossible simply because the electric field very near to the plane is very small, so that whatever structure we place here, it will be weakly excited. However, as we will show in this paper, there can exist a possibility for a very strong resonance response from an array of small particles positioned very near to an ideally conducting surface due to very strong interaction between the array particles and the ground plane. In other words, we can describe this situation using the image principle and say that the reactive impedance induced in the array particles by the image currents (plus the mutual impedance from all the other particles in the original array) can come into resonance with the proper impedance of the inclusions, if the array is positioned very close to the ground plane and the distance to the image sources is very small.

\section{REFLECTION FROM A DIPOLE ARRAY NEAR A METAL PLANE}

Let us consider a regular plane square array with period $a$ formed by passive lossless scatterers located near an ideally conducting plane and parallel to that plane. The distance between the array plane and the 


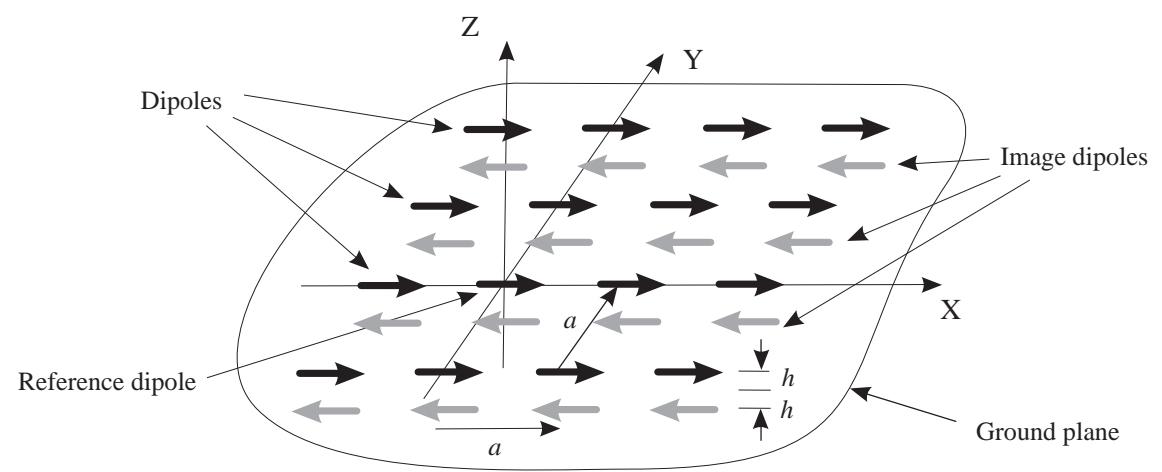

Figure 1. Geometry of the screen.

ground plane is $h$. Reflection of a plane electromagnetic wave (with electric field amplitude $\mathbf{E}_{\mathrm{inc}}$, wave vector $\mathbf{k}$ and frequency $\omega$ ) from this structure is considered. We assume the normal plane-wave incidence, to simplify the analysis. The geometry is illustrated in Figure 1.

Let us model the scatterers by electric dipoles. In the other words, let us use the dipole approximation and the local-field approach: the electric field produced by each scatterer at the centers of the adjacent ones is assumed to be equal to the field of an electric dipole and the local field distribution inside the scatterer volume is assumed to be uniform. Assuming the contribution of other multipoles to be negligible we restrict our theory by the domain $k l \ll 1$, where $l$ is the characteristic size of the inclusion. Simultaneously, we neglect the variation of the local field over the scatterer's volume. The dipole assumption simplifies the problem since one does not need to include into consideration the parameters of geometry and material of scatterers. The only value that characterizes the scatterers is their time-harmonic polarizability $\alpha(\omega)$.

The interaction of the grid with the ideally conducting ground plane can be modeled using the image theory. The electric field of charges (induced on the surface of the ground plane) corresponding to each scatterer with dipole moment $\mathbf{p}$ can be expressed as the field of an image dipole $\mathbf{p}^{\prime}=-\mathbf{p}$. Let us choose the coordinate system with the zero-point in the center of one (reference) scatterer, with the $O Z$-axis normal to the ground plane and $O X, O Y$-axes parallel to the sides of the elementary cell as at Figure 1 . In this coordinate system the radius vectors of the centers of scatterers with numbers $m, n$ can be written as $\mathbf{R}_{m, n}=(m \mathbf{x}+n \mathbf{y}) a$.

Our main goal here is to show that this structure can have a resonance of the reflection coefficient for very small heights $h$, where 
it becomes a magnetic screen (reflection coefficient equal to +1 ), and find the parameters of this resonance (resonance frequency and curve width).

\subsection{Reflection Theory}

Let us write a formula for the reference particle dipole moment in terms of the local (acting on this dipole) electric field produced by the external field of the incident wave together with the fields of the other dipoles of the structure. This will lead to a self-consistent system of equations because the dipole moments of all the dipoles are equal (in the normal incidence case which we consider). This approach is the same as that presented in [4] for planar regular arrays of bianisotropic particles in free space.

The equation for the reference dipole moment can be written in the form:

$$
\alpha^{-1} p=E_{\mathrm{ext}}+E_{p^{\prime}}+\sum_{(m, n) \neq(0,0)}\left[E_{p_{m, n}}+E_{p_{m, n}^{\prime}}\right]
$$

where $p$ is the reference scatterer's dipole moment, $E_{\text {ext }}$ is the external (to the grid) electric field, $E_{p^{\prime}}$ is the field of the reference scatterer's image dipole, $E_{p_{m, n}}$ and $E_{p_{m, n}^{\prime}}$ are the fields of the other scatterers and their image dipoles, respectively. The external (to the grid) field is formed by the incident plane wave field and the field of the incident wave reflected from the metal plane:

$$
E_{\text {ext }}=2 j \sin (k h) E_{\text {inc }}
$$

where $k=\omega \sqrt{\varepsilon_{0} \mu_{0}}$. The electric fields of the scatterers and the image dipoles can be expressed through the dipole Green function $G(\omega, \mathbf{R})$ :

$$
E_{p}(\mathbf{R})=G(\omega, \mathbf{R}) p
$$

The explicit expression for $G(\omega, \mathbf{R})$ is the following:

$$
\begin{aligned}
\overline{\bar{G}}(\omega, \mathbf{R}(x, y, z))= & \frac{k^{2}}{4 \pi \varepsilon_{0}} \frac{e^{-j k R}}{R^{3}} \\
& \cdot\left[\left(y^{2}+z^{2}\right)+\left(\frac{j}{k R}+\frac{1}{k^{2} R^{2}}\right)\left(2 x^{2}-y^{2}-z^{2}\right)\right]
\end{aligned}
$$

where $x, y, z$ are the coordinates of dipole $p$.

We consider the normal incidence, which means that all the dipole moments of scatterers are equal $p_{m, n}=p$. Thus, Equation (1) can be 
rewritten as:

$$
\alpha^{-1} p=E_{\text {ext }}+\left\{-G\left(2 h \mathbf{z}_{0}\right)+\sum_{(m, n) \neq(0,0)}\left[G\left(\mathbf{R}_{m, n}\right)-G\left(\mathbf{R}_{m, n}+2 h \mathbf{z}_{0}\right)\right]\right\} p
$$

where $\mathbf{z}_{0}$ is the unit vector along $O Z$-axis. Let us denote by $C$ the expression in the brackets in the previous formula:

$$
C=-G\left(2 h \mathbf{z}_{0}\right)+\sum_{(m, n) \neq(0,0)}\left[G\left(\mathbf{R}_{m, n}\right)-G\left(\mathbf{R}_{m, n}+2 h \mathbf{z}_{0}\right)\right]
$$

Calculation of this function (we will call it interaction constant) is an enough complex problem (see below), but if it is solved, we get the solution of the excitation problem:

$$
p=2 j \sin (k h)\left(\alpha^{-1}-C\right)^{-1} E_{\text {inc }}
$$

The reflection coefficient from the structure is equal to

$$
R=-1+\frac{k}{\varepsilon_{0} a^{2}} \frac{2 j \sin ^{2}(k h)}{\alpha^{-1}-C}
$$

This relation can be obtained as the zero term of the classical Floquet representation for the field of the grids (original grid and the image one) using Expression (7) for determination of the scatterer's dipole moment. This way we can easily calculate the reflection coefficient using Formula (8), if we know how to calculate the dynamic interaction constant $C$.

Let us note that for small heights $h$ the reflection coefficient formula contains a very small value of sine function of $k h$ squared. Two physical phenomena are taken into account by this term. The first one is that acting to the grid external field is the sum of the incident wave and the corresponding reflected wave from the ground plane. The second one is that the plane wave produced by the grid also consists of the original wave and that reflected from the ground plane (this second term can be associated with the field produced by the grid of the image dipoles).

\subsection{Interaction Constant}

The first term of the expression for the interaction constant (6) corresponds to the image dipole of the zero scatterer. The second term is formed by the series of the dyadic Green's functions for the original 
and image grids. These series are non-convergent in the classical meaning and must be calculated using special methods. It is also possible to make this summation analytically.

Let us split the series of dipole Green's functions for the original and image grids in (6) and denote the terms as $\beta$ and $\gamma$, respectively:

$$
\beta=\sum_{(m, n) \neq(0,0)} G\left(\mathbf{R}_{m, n}\right), \quad \gamma=\sum_{m, n} G\left(\mathbf{R}_{m, n}+2 h \mathbf{z}_{0}\right), \quad C=\beta-\gamma
$$

Constant $\beta$ is known as key dyadic [4] and as interaction constant [6] of a single two-dimensional square grid in free space. It can be calculated by splitting of the series into wave and quasi-static terms [4] or using the Poisson summation formula with singularity cancellation [6]. Constant $\gamma$ is formed by the series corresponding to the image grid and can be calculated using the Floquet representation [7].

For dense grids (more precisely, if $k a<1$ ) with square cells it is preferable to use approximate formulae based on the idea of averaging $[5,8,9]$ for calculation of interaction constants $\beta$ and $\gamma$ :

$$
\begin{aligned}
& \beta= \frac{k}{4 \varepsilon_{0} a^{2}}\left(\frac{\cos k R_{0}}{k R_{0}}-\sin k R_{0}\right)+j\left[\frac{k^{3}}{6 \pi \varepsilon_{0}}-\frac{k}{2 \varepsilon_{0} a^{2}}\right] \\
& \gamma=\frac{k}{4 \varepsilon_{0} a^{2}} {\left[\frac{R_{0}^{2}}{R_{0}^{2}+(2 h)^{2}} \frac{\cos k \sqrt{R_{0}^{2}+(2 h)^{2}}}{k \sqrt{R_{0}^{2}+(2 h)^{2}}}\right.} \\
&-\left.\left(1+\frac{(2 h)^{2}}{R_{0}^{2}+(2 h)^{2}}\right) \sin k \sqrt{R_{0}^{2}+(2 h)^{2}}\right] \\
&- \frac{1}{4 \pi \varepsilon_{0}}\left[\left(\frac{1}{(2 h)^{3}}-\frac{k^{2}}{2 h}\right) \cos 2 k h+\frac{k}{(2 h)^{2}} \sin 2 k h\right] \\
&-j \frac{k}{2 \varepsilon_{0} a^{2}} \cos 2 k h
\end{aligned}
$$

Here, parameter $R_{0}=a / 1.438$. In this model of regular dipole arrays, square-cell arrays of dipoles are replaced by a homogenized sheet of averaged polarization, which has a hole around the reference particle of the equivalent radius $R_{0}$. As has been shown in [5, 8], the approximation is valid in the frequency region $k a<1$, in the static limit $k a \rightarrow 0$ formula (10) gives the known result $\left.\beta\right|_{\omega=0}=0.3595[6$, p. 760], and in the region $0<k a<1$ (10) very well approximates the accurate solution [6, p. 784]. Expression (11) has the same region of validity [9]. In fact, the imaginary parts of the interaction constants in (10) and (11) are exact expressions [5, 9]. The imaginary 


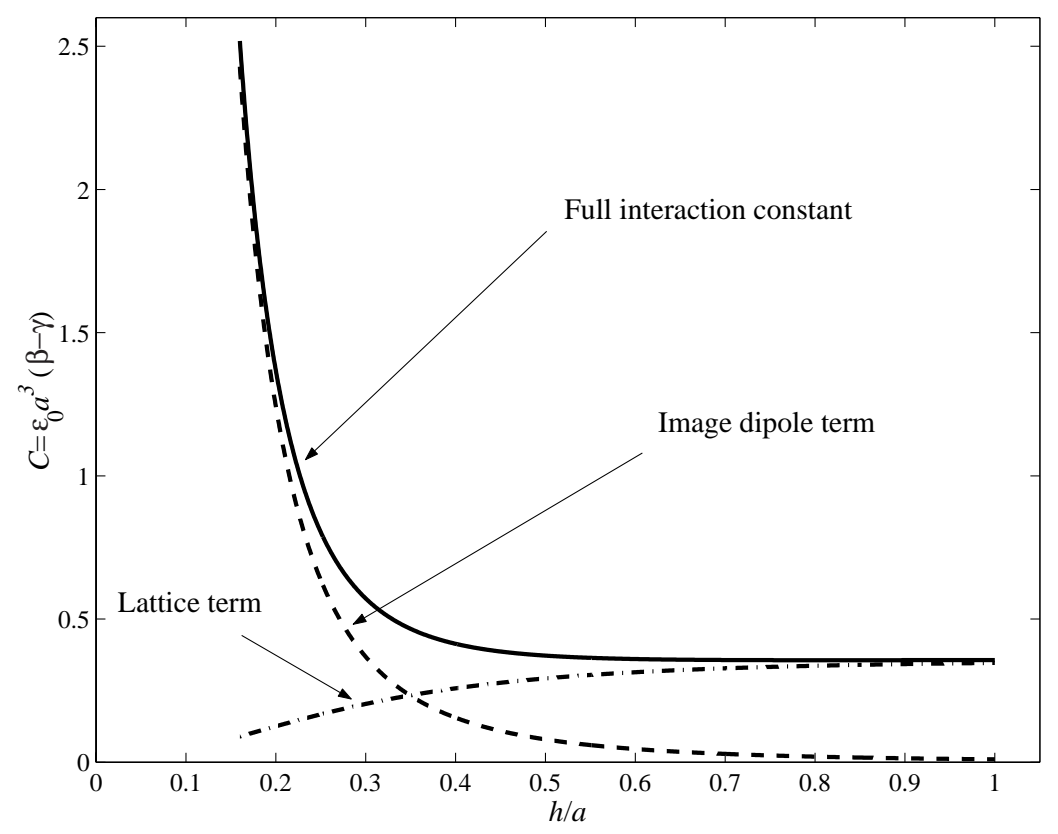

Figure 2. Interaction constant for an array of point dipoles parallel to an ideally conducting plane as functions of the normalized height $h / a$.

part of $\beta$ contains two terms: a plane-wave contribution from the averaged polarization (proportional to $\omega$ ), and a contribution which is related to single-dipole radiation (proportional to $\omega^{3}$ ). The real part corresponds to non-propagating modes existing near the array plane because the polarization is non-homogeneous. The imaginary part of $\gamma$ gives the real part of the propagating plane wave field excited by the averaged polarization of the array (the averaged surface current density is $\mathbf{J}=j \omega \mathbf{p} / a^{2}, a^{2}$ is the cell area).

Since in this study we are interested in the situation when all the sizes are very small compared to the wavelength $(k a \ll 1)$, we can use the corresponding quasi-static approximations:

$$
\begin{aligned}
& \beta=\frac{1}{4 \varepsilon_{0} a^{2} R_{0}}+j\left[\frac{k^{3}}{6 \pi \varepsilon_{0}}-\frac{k}{2 \varepsilon_{0} a^{2}}\right] \\
& \gamma=\frac{1}{4 \varepsilon_{0} a^{2}} \frac{R_{0}^{2}}{\left(R_{0}^{2}+(2 h)^{2}\right)^{3 / 2}}-\frac{1}{4 \pi \varepsilon_{0}(2 h)^{3}}-j \frac{k}{2 \varepsilon_{0} a^{2}} \cos 2 k h
\end{aligned}
$$


Thus,

$$
\begin{aligned}
C= & \beta-\gamma \\
= & \frac{1}{4 \varepsilon_{0} a^{2} R_{0}}\left[1-\frac{1}{\left[1+\left(2 h / R_{0}\right)^{2}\right]^{3 / 2}}\right]+\frac{1}{4 \pi \varepsilon_{0}(2 h)^{3}} \\
& +j\left[\frac{k^{3}}{6 \pi \varepsilon_{0}}-\frac{k}{\varepsilon_{0} a^{2}} \sin ^{2}(k h)\right]
\end{aligned}
$$

For lossless particles, the imaginary part of $\alpha^{-1}$ is determined by its radiation resistance only $[5]$ :

$$
\operatorname{Im}\left\{\alpha^{-1}\right\}=\frac{k^{3}}{6 \pi \varepsilon_{0}}
$$

and the reflection coefficient (8) can be rewritten upon substitution of the exact imaginary part of the interaction constant $C$ as

$$
R=-1+\frac{2}{1-j \varepsilon_{0} a^{2} \frac{\operatorname{Re}\left\{\alpha^{-1}-C\right\}}{k \sin ^{2} k h}}
$$

This formula clearly shows the energy balance: in this case the structure is lossless and of course the absolute value of the reflection coefficient is equal to unity: $|R|=1$.

\section{RESONANCE CONDITION}

\subsection{Point Dipoles}

From (14) we observe that the real part of quantity $C$ is positive. The real part of the inverse polarizability $\alpha^{-1}$ for small conducting particles is also positive, so in principle $\operatorname{Re}\{C\}$ can match the positive real part of $\alpha^{-1}$. The problem is that if the polarizability value is small, its inverse value is large, and it cannot be compensated by the interaction constant. In this case the reflection coefficient is close to -1 , which corresponds to an electric screen. For higher polarizabilities the resonance is possible when $\operatorname{Re}\left\{\alpha^{-1}-C\right\}=0$. The reflection coefficient becomes equal to +1 which corresponds to a magnetic screen.

First we observe that for point-dipole particles the resonance at small heights is always possible, for arbitrary particle polarizabilities. Indeed, the near field of the nearest image dipole (which is proportional to $1 / h^{3}$ ) dominates at very small heights, see (14), and in the limit $h \rightarrow 0$ we have $\operatorname{Re}\{C\} \rightarrow \infty$. This means that for arbitary small 
polarizabilities $\alpha$ there exists a point where the resonance condition $\operatorname{Re}\left\{\alpha^{-1}\right\}=\operatorname{Re}\{C\}$ is satisfied. This can be seen from Figure 2, where we illustrate the behavior of the interaction constant as dependent on the distance to the ground plane. However, this reasoning assumes zero size of the particles.

\subsection{Spherical Particles}

Let us next consider dielectric spherical scatterers with relative permittivity $\varepsilon_{r}$ and for simplicity choose a special small value of $h=a$. In this special case, assuming $k a \ll 1$,

$$
\operatorname{Re}\{\beta-\gamma\}=\frac{0.4263}{\varepsilon_{0} a^{3}}
$$

Next, let us substitute the sphere polarizability

$$
\alpha=4 \pi \varepsilon_{0} r^{3} \frac{\varepsilon_{r}-1}{\varepsilon_{r}+2}
$$

where $r$ is the sphere radius, and check if the resonance condition

$$
\frac{1}{\alpha}=\operatorname{Re}\{\beta-\gamma\}
$$

can be satisfied. The solution of equation (19) reads

$$
\frac{a^{3}}{r^{3}}=5.3576 \frac{\varepsilon_{r}-1}{\varepsilon_{r}+2}
$$

For $\varepsilon_{r}>1$, this solution gives always $\frac{a^{3}}{r^{3}}<5.3576$ or $a<1.7498 r$. However, spheres touch each other at $a=2 r$. Thus, no resonance is possible, and one can only expect that rather strong response can be realized if the packaging is dense and the distance to the ground is small.

One can suggest to use planar particles, like thin strips, so that the interaction constant can be increased by choosing a very small distance to the ground, smaller than the particle size. However, in this case the previous estimations for the interaction constant which showed unlimited increase of $\gamma$ with decreasing the height are not valid. This is because in this case the near field of the mirror-image particle is not the point-source field. This problem can be easily studied numerically, but the result is anyway easy to predict. Taking for simplicity narrow strip particles, we observe that $\operatorname{Re}\left\{\alpha^{-1}\right\}$ is proportional to the imaginary part of the input impedance of a single strip considered as a dipole 
antenna. At very small heights, the interaction field is mainly the field created by the nearest image particle. Thus, the value of $\operatorname{Re}\{\beta-\gamma\}$ is proportional to the imaginary part of the mutual impedance between the original strip and its mirrow image. Since the last quantity is always smaller than the proper impedance, we conclude that the resonance is not actually possible. This conclusion is quite general and is valid for arbitary shapes of inclusions.

From these considerations we see that to realize very thin resonant layers we should find a way to increase the particle polarizability without increasing its size (since we should position the array very near to the ground plane to provide a large value of the interaction constant).

\subsection{Arrays of Short Loaded Wire Antennas}

Here we suggest that this can be made possible by loading particles (short strip dipoles with capacitive input impedance) by bulk loads. If the load impedance is inductive, the particle polarizability near the particle resonance becomes large, which makes it possible to fulfill the resonance condition for a grid over conducting plane.

Suppose that the array consists of short wire dipoles with total length $2 l$ loaded by bulk reactive impedances $Z$. For our purpose, the load impedance should be inductive, so we denote $Z=j \omega L$. Using the antenna model [10] for determination of the polarizability of loaded wires (for the real part of the polarizability's inverse value) we have:

$$
\alpha=\left(\frac{C_{\text {wire }}^{-1}-\omega^{2} L}{l^{2}}+j \frac{k^{3}}{6 \pi \varepsilon_{0}}\right)^{-1}
$$

where $C_{\text {wire }}=\pi l \varepsilon_{0} / \log \left(2 l / r_{0}\right)$ is the capacitance of the wire, $L$ is the inductance of the load, $l$ is the half length of wire and $r_{0}$ is the wire radius.

It is more convenient to analyze loaded arrays rewriting the reflection coefficient expression (using (16) and (21) for $k h \ll 1$ ) as

$$
R=-1+\frac{2}{1-j \eta a^{2} \frac{\left(C_{\text {wire }}^{-1}-C l^{2}\right)-\omega^{2} L}{\omega^{3}\left(\mu_{0} h l\right)^{2}}}
$$

where $\eta=\sqrt{\mu_{0} / \varepsilon_{0}}$. We can see that indeed there is a resonance of the reflection coefficient with the central frequency

$$
\omega_{0}=\sqrt{\frac{C_{\text {wire }}^{-1}-C l^{2}}{L}}
$$




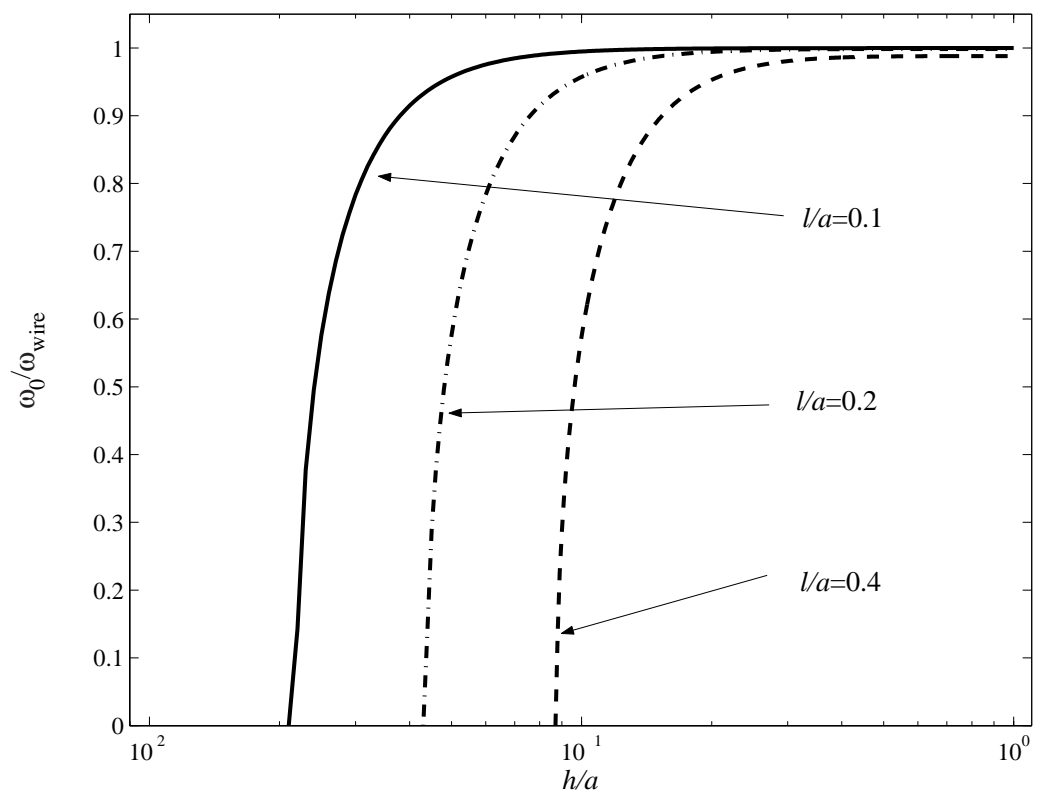

Figure 3. Dependence of the resonance frequency shift on the normalized thickness of the screen. In this example $l / r_{0}=10$.

It is important to note that this value is smaller than the resonance frequency of individual loaded wires $\omega_{\text {wire }}=1 / \sqrt{L C_{\text {wire }}}$ due to the field interaction between inclusions. The dependence of the resonance frequency shift $\omega_{0} / \omega_{\text {wire }}$ on the thickness of the screen (calculated by formula (23)) is plotted on Fig. 3. When the height is large, the resonance is determined by the properties of the scatterer, with a small shift due to interactions within the array. At small heights, the field of the image dipole becomes large, and the resonance frequency of the reflection coefficient $\omega_{0}$ becomes much smaller than the resonance frequency of a single array element $\omega_{\text {wire }}$, and in theory the resonance frequency can approach zero. In practice this is restricted by the particle size.

Finally, let us consider the resonance curve width. This can be found from the following condition:

$$
\left.\varepsilon_{0} a^{2} \frac{\operatorname{Re}\left\{\alpha^{-1}-C\right\}}{k \sin ^{2} k h}\right|_{\omega_{0}+\Delta \omega / 2}=-1
$$




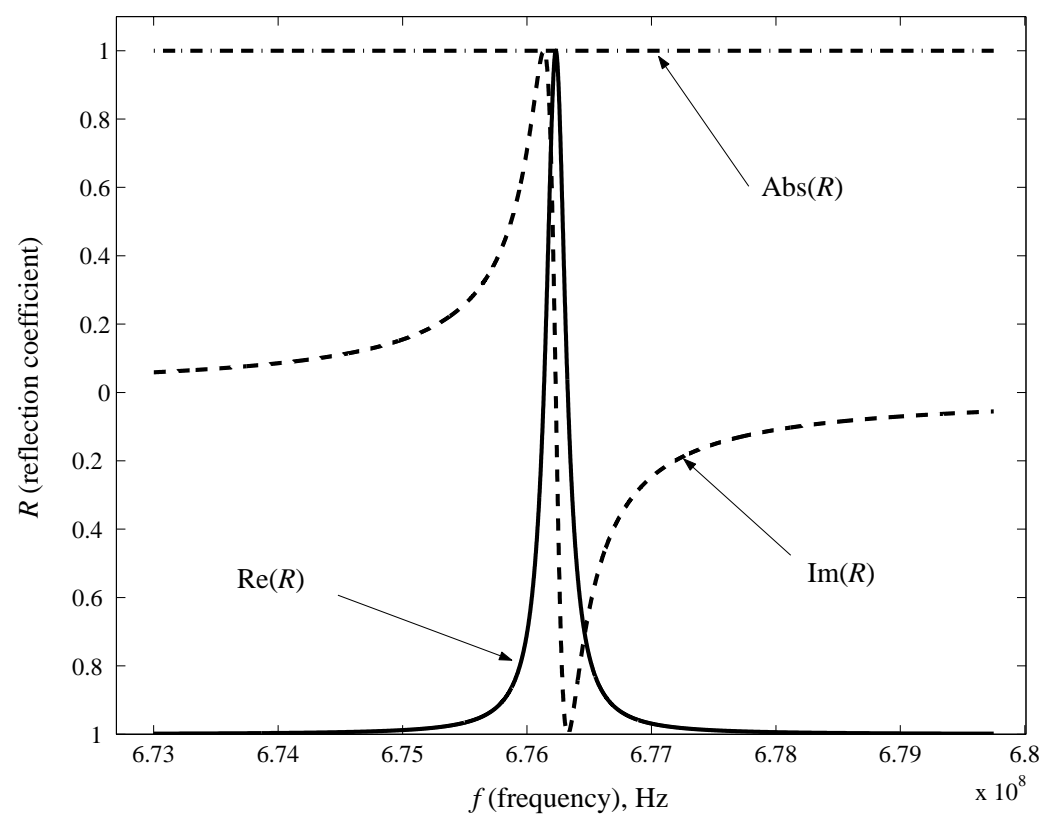

Figure 4. Dependence of the reflection coefficient on the frequency for $1 \mathrm{~cm}$ square grid of $8 \mathrm{~mm}$ wires loaded by $1 \mathrm{nH}$ bulk inductances positioned at $1 \mathrm{~cm}$ height over a metal plane.

(see Equation (16)). From here, assuming $\Delta \omega \ll \omega_{0}$, we find

$$
\frac{\Delta \omega}{\omega_{0}}=\mu_{0} \frac{k(l h)^{2}}{a^{2} L}
$$

Further, writing the load inductance $L=\mu_{0} d$, where $d$ is the characteristic size of the coil, we can express this as

$$
\frac{\Delta \omega}{\omega_{0}}=\frac{l^{2}}{a^{2}} \frac{k h^{2}}{d}
$$

Thus, the resonance curve width is proportional to the height squared and to the resonance frequency. The resonance curve width is larger for dense grids and for high frequencies. It is important to note that for very small heights $h$ the resonance becomes very sharp.

\section{NUMERICAL EXAMPLE}

Calculations of the reflection coefficient have been made for a structure with the following parameters: a dipole grid with square $1 \mathrm{~cm}$ 


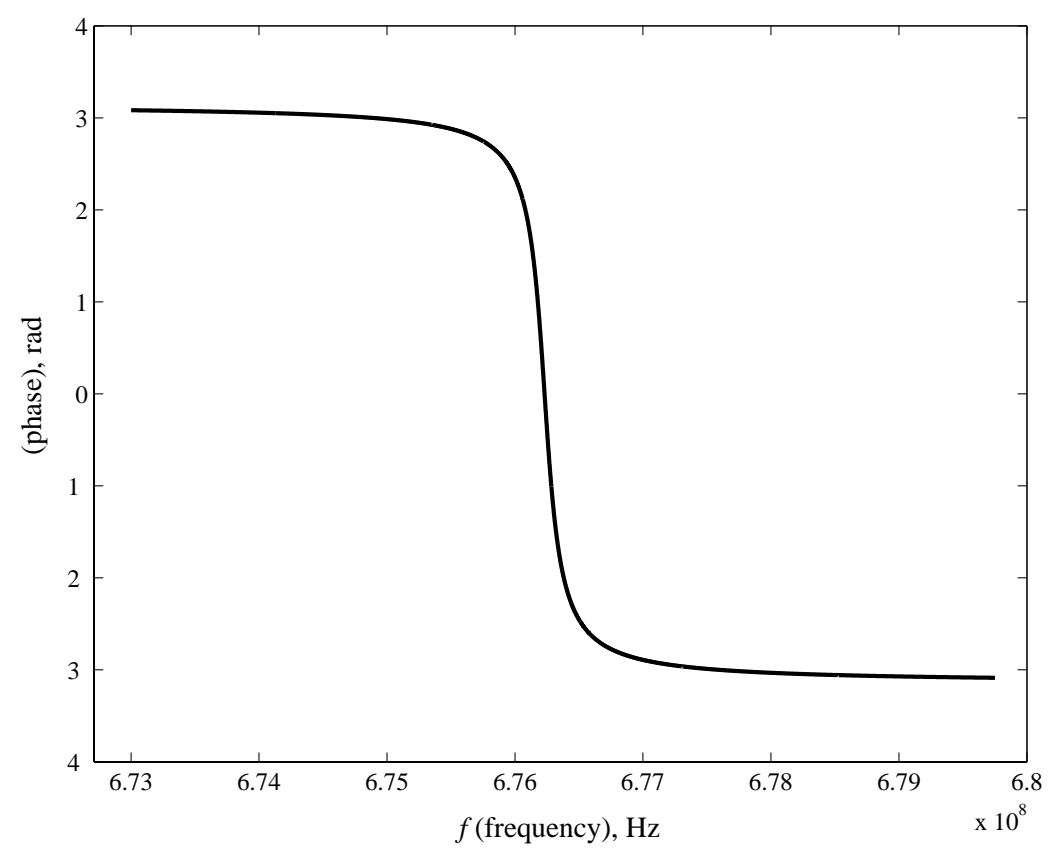

Figure 5. Dependence of the phase shift on the frequency.

elementary cell $(a=b=1 \mathrm{~cm})$ formed by wires of the total length $8 \mathrm{~mm}(l=4 \mathrm{~mm})$ and $1 \mathrm{~mm}$ radius $\left(r_{0}=1 \mathrm{~mm}\right)$ loaded by inductances $L=1 \mathrm{nH}$. Estimations show that such inductance can be realized by a coil structure of characteristic size about $5 \mathrm{~mm}$, so this value is realistic. This grid is positioned at $1 \mathrm{~cm}$ height $(h=1 \mathrm{~cm})$ over an ideally conducting plane. The normal incidence is under consideration. The dependence of the reflection coefficient (its real and imaginary parts) on the frequency is presented on Fig. 4. The phase shift of the reflected wave dependence on the frequency is shown on Fig. 5.

The resonance frequency of the loaded wires corresponds to 688.2 $\mathrm{MHz}\left(\omega_{\text {wire }}=4.324 \mathrm{GHz} \cdot \mathrm{rad}\right)$. The resonance of the grid appears at about $676.2 \mathrm{MHz}\left(\omega_{0}=4.249 \mathrm{GHz} \cdot \mathrm{rad}\right)$. The thickness of the "covering" in this example is rather small indeed: $h / \lambda \approx 0.02$. We observe a very narrow resonance with the width of about $190 \mathrm{kHz}$ $\left(\Delta \omega_{0} / \omega_{0}=0.28 \cdot 10^{-3}\right)$. 


\section{CONCLUSION}

In this paper, we have proposed a method to realize extremely thin resonant coverings of ideally conducting bodies which can provide resonance response and realize artificial magnetic surfaces. It has been shown that this goal can be achieved using regular arrays of small dipole antennas loaded by bulk inductive loads.

Such two-dimensional regular grids of loaded wires placed over an ideally conducting plane have been studied in detail. A simple analytical theory for plane electromagnetic wave reflection from this structure has been presented for the normal incidence. From this theory it follows that the structure possesses a narrow resonance of the reflection coefficient. At resonance the reflection coefficient from the grid equals to +1 . Thus, this structure behaves as a magnetic screen near the resonance, instead of an electric one as for frequencies far from the resonance. Analytical formulae for calculation of the resonance frequency and the resonance curve width are given. It turns out that this resonance frequency is smaller than the loaded wires resonant frequency, due to field interactions between the particles and the ground plane. Numerical calculations of the reflection coefficient for a structure with some realistic parameters have been done and dependencies of the reflection coefficient and its phase on the frequency have been plotted.

\section{REFERENCES}

1. Sievenpiper, D., L. Zhang, R. F. J. Broas, N. G. Alexopoulos, and E. Yablonovich, "High-impedance electromagnetic surfaces with a forbidden frequency band," IEEE Trans. Microwave Theory Techniques, Vol. 47, 2059-2074, 1999.

2. Usanov, D. A., S. S. Gorbatov, V. E. Orlov, and S. B. Wenig, "Resonances in a semiinfinite waveguide with iris in relation to the excitation of higher-order modes," Technical Physics Lett., Vol. 26, No. 9, 827-828, 2000.

3. Terracher, F. and G. Berginc, "Scattering by doubly periodic gaps and cracks," J. Electromagn. Waves Applic., Vol. 14, No. 8, 11431158, 2000.

4. Simovski, C. R., M. S. Kondratjev, P. A. Belov, and S. A. Tretyakov, "Interaction effects in two-dimensional bianisotropic arrays," IEEE Trans. Antennas Propagat., Vol. 47, No. 9, 1429-1439, 1999.

5. Tretyakov, S. A., A. J. Viitanen, S. I. Maslovski, and I. E. Saarela, "Impedance boundary conditions for regular dense arrays of dipole 
scatteres," Electromagnetics Laboratory Report Series, Helsinki University of Technology, Report 304, August 1999, submitted to IEEE Transactions Antennas Propagation.

6. Collin, R. E., Field Theory of Guided Waves, 2nd ed., IEEE Press, 1991.

7. Simovski, C. R., P. A. Belov, and M. S. Kondratjev, "Electromagnetic interaction of chiral particles in three dimensional arrays," J. Electromagn. Waves Applics., Vol. 13, 189-204, 1999.

8. Maslovski, S. I. and S. A. Tretyakov, "Full-wave interaction field in two-dimensional arrays of dipole scatterers," Int. J. Electron. Commun. (AË̈), Vol. 53, No. 3, 135-139, 1999.

9. Yatsenko, V., S. Maslovski, and S. Tretyakov, "Electromagnetic interaction of parallel arrays of dipole scatterers," Progress in Electromagnetics Research, PIER25, 285-307, 2000 (Abstract also in J. Electromagnetic Waves Applic., Vol. 14, 79-80, 2000).

10. Tretyakov, S. A., F. Mariotte, C. R. Simovski, T. G. Kharina, and J.-Ph. Heliot, "Analytical antenna model for chiral scatterers: comparison with numerical and experimental data," IEEE Trans. Antennas Propagat., Vol. 44, No. 7, 1006-1014, 1996.

Pavel A. Belov received the B.Sc. and M.Sc. in mathematics from the St. Petersburg Institute of Fine Mechanic and Optics (Russia) in 1998 and 2000, respectively. Since 2000 he has been Ph.D. student of the Physics Department of the St. Petersburg Institute of Fine Mechanic and Optics. Since 2001 he has been researcher and PhD student in the Radio Laboratory, Helsinki University of Technology (Finland). He received 1999 and 2000 SPIE Scolarships, 2001 IEEE MTT-S Fellowship, and 2001 INTAS Fellowship. His scientific interests include analytical and numerical modeling of electromagnetic properties of photonic crystals, artificial microwave periodic structures, composite materials and high impedance surfaces.

Sergei A. Tretyakov received the Dipl. Engineer-Physicist, the Ph.D., and the Doctor of Sciences degrees (all in radiophysics) from the St. Petersburg State Technical University (Russia), in 1980, 1987, and 1995, respectively. From 1980 to 2000 he was with the Radiophysics Department of the St. Petersburg State Technical University. Presently, he is professor of radio engineering in the Radio Laboratory, Helsinki University of Technology. His main scientific interests are electromagnetic field theory, complex media electromagnetics and microwave engineering. Prof. Tretyakov served as Chairman of the St. Petersburg IEEE ED/MTT/AP Chapter from 1995 to 1998. 